\title{
Control óptimo determinista aplicado al problema económico de crecimiento endógeno
}

\author{
V.M. García-Guerrero y F. Venegas-Martínez \\ División de Estudios de Posgrado de la Facultad de Ingeniería, UNAM y \\ Centro de Investigación en Finanzas, Tecnológico de Monterrey, Campus Ciudad de M éxico. \\ E-mails: vmgarciag23@yahoo.com.mx y fvenegas@itesm.mx
}

(recibido: junio de 2004; aceptado: septiembre de 2004)

\begin{abstract}
Resumen
El propósito de este trabajo consiste en desarrollar, dentro de un marco determinista, un modelo de crecimiento económico que explique endógenamente los determinantes del mismo. Asimismo, el modelo propuesto describe el comportamiento de la dinámica de las trayectorias de consumo y capital de los agentes. Se supone que la economía está poblada por individuos idénticos con vida infinita que desean maximizar su utilidad por un bien de consumo perecedero. Distintos índices de satisfacción se revisan. Las empresas comparten la misma tecnología. En dicha tecnología, el producto marginal del capital se mantiene constante en el tiempo. En este contexto, el problema de decisión de un consumidor representativo se plantea como un problema de control óptimo determinista en tiempo continuo. Las decisiones óptimas del consumidor se examinan utilizando subrutinas de MATLABO .
\end{abstract}

Descriptores: Control óptimo, optimización dinámica, crecimiento endógeno.

\begin{abstract}
Theaim of this pa per is to develop, in a deter min isticframework, a model of eco nomic growth that explainsits deter mi nants en dog enously. Theproposed model also describes thebehavior of thedy namics of theagent's con sump tion and cap i tal paths. It is sup posed that theecon omy is pop u lated by identi cal and in fi nitely lived in di vidu als that wish to maxi mizetheir util ity for thecon sumption of a sin gle perish ablegood. Several sat isfaction in dexes arerevised. Firms sharethesametech nol ogy. In such a technol ogy it is as sumed that the mar ginal prod uct of cap i tal remains con stant through time. In this context, thecon sumer's deci sion problem is stated as a deter min isticop ti mal con trol probem in con tin u ous time. Con sumer's op ti mal deci sionsareex am ined by us ing M A TLA B @ subroutines.
\end{abstract}

Keywords: $0 \mathrm{pti}$ mal con trol, dy namicop ti mi za tion, en dogen ousgrowth.

\section{Introducción}

En la actualidad, la búsqueda de soluciones a los problemas económicos requiere de un planteamiento multidisciplinario que integre diversas áreas del conocimiento científico. En particular, el análisis financiero es la disciplina que más se ha combinado con la economía en el modelado del comportamiento de los agentes y de los mercados en que estos participan.
Asimismo, entre las herramientas matemáticas que se utilizan para resolver muchos de los problemas económico-financieros destaca la optimización dinámica, la cual agrega las técnicas del cálculo de variaciones, el control óptimo y la programación dinámica.

En este trabajo se desarrolla un modelo de crecimiento endógeno balanceado que permite analizar las decisiones óptimas de los agentes 
bajo diferentes funciones de utilidad. Los supuestos del modelo se mantienen tan simples como sea posible, de tal manera que se puedan obtener soluciones analíticas. En esta investigación, también se estudia la evolución del consumo y el cap ital en función de la tasa subjetiva de descuento y del producto marginal de capital. Asimismo, se analiza la evolución del consumo y del capital en función del tiempo.

La estructura de este trabajo se encuentra organizada de la siguiente manera. En la sección 2, se presenta el Hamiltoniano en valor presente, uno de los conceptos importantes en el planteamiento matemático del problema de crecimiento. En el transcurso de la sección 3 , se plantean los problemas de optimización asociados con un consumidor racional con vida infinita que desea maximizar su utilidad por un bien de consumo perecedero. Dichos problemas se resuelven con con trol óptimo determinista en tiempo continuo. Asimismo, las decisiones óptimas del consumidor se examinan utilizando subrutinas de MATLAB $\odot$. Por último, en la sección 4 se establecen las conclusiones y algunas limitaciones.

\section{EI Hamiltoniano en valor presente}

Existen muchos problemas económicos que se pueden plantear como problemas de control óptimo. En la mayoría de ellos el funcional objetivo es una in te gral, en cuyo integrando aparece un fac tor de descuento,

$$
e^{-p t}
$$

donde $\rho$ es una tasa de descuento. Asimismo, la restricción de estos problemas es una ecuación diferencial. En este marco, se plantea el siguiente problema:

$$
\begin{aligned}
\text { Maximizar } J\left(\mathrm{k}_{\mathrm{t}}\right) & =\int_{0}^{\infty} F\left(\mathrm{k}_{\mathrm{t}}, \mathrm{c}_{\mathrm{t}}\right) \mathrm{e}^{-p \mathrm{t}} \mathrm{dt} \\
\text { sujeto a } \quad & \dot{\mathrm{k}}_{\mathrm{t}}=\mathrm{G}\left(\mathrm{k}_{\mathrm{t}}, \mathrm{c}_{\mathrm{t}}\right), \\
& \mathrm{k}_{0} \text { constante, } \\
& \mathrm{c}_{\mathrm{t}} \in \Omega,
\end{aligned}
$$

donde $c_{\mathrm{t}}$ es la vari able de con trol y $\mathrm{k}_{\mathrm{t}}$ es la vari able de estado. El Hamiltoniano del problema anterior se define como

$$
H\left(\mathrm{k}_{\mathrm{t}}, \mathrm{c}_{\mathrm{t}}, \mathrm{m}_{\mathrm{t}}\right)=F\left(\mathrm{k}_{\mathrm{t}}, \mathrm{c}_{\mathrm{t}}\right) \mathrm{e}^{-\rho \mathrm{t}}+\mathrm{m}_{\mathrm{t}} G\left(\mathrm{k}_{\mathrm{t}}, \mathrm{c}_{\mathrm{t}}\right)
$$

donde $m_{t}$ es el multiplicador de Lagrange asociado a la restricción. Ahora bien, las condiciones necesarias de un máximo están dadas por

$$
\begin{aligned}
\frac{\partial H}{\partial \mathrm{c}_{\mathrm{t}}} & =0 \\
-\frac{\partial H}{\partial \mathrm{k}_{\mathrm{t}}} & =-\frac{\partial F}{\partial \mathrm{k}_{\mathrm{t}}} \mathrm{e}^{-\rho \mathrm{t}}-\mathrm{m}_{\mathrm{t}} \frac{\partial G}{\partial \mathrm{k}_{\mathrm{t}}} \\
\frac{\partial H}{\partial \mathrm{m}_{\mathrm{t}}} & =G\left(\mathrm{k}_{\mathrm{t}}, \mathrm{c}_{\mathrm{t}}\right) .
\end{aligned}
$$

El Hamiltoniano en valor presente se define de la siguiente manera:

$$
\mathrm{H}=\mathrm{He}^{\rho \mathrm{t}}=F\left(\mathrm{k}_{\mathrm{t}}, \mathrm{C}_{\mathrm{t}}\right)+\mathrm{m}_{\mathrm{t}} \mathrm{e}^{\rho \mathrm{t}} G\left(\mathrm{k}_{\mathrm{t}}, \mathrm{C}_{\mathrm{t}}\right) .
$$

Si se denotam ${ }_{t}=\lambda_{t} e^{-\phi}$, la ecuación an te rior se puede reescribir de la siguiente manera:

$$
\begin{gathered}
\mathrm{H}\left(\mathrm{k}_{\mathrm{t}}, \mathrm{c}_{\mathrm{t}}, \lambda_{\mathrm{t}}\right) . \\
=F\left(\mathrm{k}_{\mathrm{t}}, \mathrm{c}_{\mathrm{t}}\right)+\lambda_{\mathrm{t}} G\left(\mathrm{k}_{\mathrm{t}}, \mathrm{c}_{\mathrm{t}}\right) .
\end{gathered}
$$

Bajo este cambio de variable, las condiciones necesarias para un máximo son ahora las siguientes

$$
\begin{aligned}
\frac{\partial \mathrm{H}}{\partial c_{\mathrm{t}}} & =0, \\
-\frac{\partial \mathrm{H}}{\partial \mathrm{k}_{\mathrm{t}}} & =\dot{\lambda}_{\mathrm{t}}-\lambda_{\mathrm{t}} \rho, \\
\frac{\partial \mathrm{H}}{\partial \lambda_{\mathrm{t}}} & =\dot{\mathrm{k}}_{\mathrm{t}}
\end{aligned}
$$

El lector interesado puede consultar Cerdá (2001) y Chiang (1992) para más detalles del principio del máximo y del Hamiltoniano en valor presente.

\section{Modelos económicos de crecimiento endógeno}

Considere una economía cerrada que pro duce y consume un bien perecedero. En este caso, la identidad de la renta nacional en términos per capita está dada por 


$$
y_{t}=c_{t}+i_{t}+g_{t}
$$

Esta condición establece que toda la producción, $y_{t}$, del bien es destinada a tres fines: al consumo, $G$, a la inversión, $i_{t}, y$ al gasto de gobierno, gt. En lo que sigue, por simplicidad, se supondrá que $g_{t}=0$. La condición (1), asegura el equilibrio en el mercado de bienes. El problema de optimización que desea resolver un consumidor racional, maximizador de utilidad, es el siguiente:

$$
\begin{aligned}
& \text { Maximizar } \int_{0}^{\infty} u\left(c_{\mathrm{c}}\right) \mathrm{e}^{-\rho \mathrm{t}} \mathrm{dt} \\
& \text { sujeto a } \mathrm{y}_{\mathrm{t}}=c_{\mathrm{t}}+\dot{\mathrm{k}}_{\mathrm{t}}, \\
& \mathrm{k}_{0} \mathrm{dado},
\end{aligned}
$$

donde $c_{t}$ es el consumo del individuo al tiempot; $k_{t}$ es el capital del individuo al tiempo $t$; $u\left(c_{t}\right)$ es la función de utilidad o satisfacción del individuo y? es la tasa subjetiva de descuento. Si se supone que la empresa representativa pro duce el bien con una tecnología de la forma $\mathrm{y}_{\mathrm{t}}=A \mathrm{k}_{\mathrm{t}}$ entonces el producto marginal de capital, $A$, se mantiene constante en el tiempo. De esta manera, el problema an te riorse transforma en

$$
\begin{aligned}
& \text { Maximizar } \int_{0}^{\infty} u\left(c_{t}\right) e^{-p t} d t \\
& \text { sujeto a } \dot{k}_{t}=A k_{t}-c_{t}, \\
& k_{0} d a d o
\end{aligned}
$$

Con base en el principio del máximo, las condiciones de primer orden (o condiciones necesarias) están dadas por:

$$
\begin{aligned}
\frac{\partial \mathrm{H}}{\partial c_{\mathrm{t}}} & =0, \\
-\frac{\partial \mathrm{H}}{\partial \mathrm{k}_{\mathrm{t}}} & =\dot{\lambda}_{\mathrm{t}}-\lambda_{\mathrm{t}} \rho, \\
\frac{\partial \mathrm{H}}{\partial \lambda_{\mathrm{t}}} & =A \mathrm{k}_{\mathrm{t}}-c_{\mathrm{t}},
\end{aligned}
$$

donde $H=H(k, c, \lambda, t)=u\left(c_{t}\right)-\lambda_{t}\left(A k_{t}-c_{t}\right)$.

\section{Función de utilidad logarítmica}

Suponga que la función de utilidad del problema (2) es la siguiente:

$$
u\left(c_{t}\right)=\ln \left(c_{t}\right)
$$

El Hamiltoniano asociado a este problema está dado por:

$$
\mathrm{H}(\mathrm{k}, \mathrm{c}, \lambda)=\ln \left(c_{\mathrm{t}}\right)+\lambda_{\mathrm{t}}\left(A \mathrm{k}_{\mathrm{t}}-\mathrm{c}_{\mathrm{t}}\right)
$$

donde $?_{t}$ es la variable de coestado. A partir de la condición (4) se sigue que

$$
\dot{\lambda}_{t}=\lambda_{t}(\rho-A)
$$

Por lo tanto,

$$
\lambda_{t}=\lambda_{0} e^{(\rho-A)^{t}}
$$

La condición (3) con duce a

$$
c_{t}=\frac{1}{\lambda_{t}}
$$

Si se sustituye el valor de ${ }_{t}$ en la ecuación an terior, se sigue que

$$
c_{t}=\frac{1}{\lambda_{0}} e^{(A-\rho)^{t}}
$$

Ahora bien, como $c_{t}=A k_{t}-\dot{K}_{t}$, se tiene que

$$
\mathrm{G}^{-A \mathrm{t}}=A \mathrm{k}_{\mathrm{t}} \mathrm{e}^{-A t}-\dot{\mathrm{k}}_{\mathrm{t}} \mathrm{e}^{-A t}
$$

Si se integra la expresión an te rior se obtiene

$$
\int_{0}^{\infty} c_{t} \mathrm{e}^{-A t} \mathrm{dt}=\int_{0}^{\infty} A k_{\mathrm{t}} \mathrm{e}^{-A t} \mathrm{dt}-\int_{0}^{\infty} \dot{\mathrm{k}}_{\mathrm{t}} \mathrm{e}^{-A \mathrm{t}} \mathrm{dt}
$$

Observe que la integración por partes del primer sumando del lado derecho de la ecuación an teriorproduce

ó

$$
\int_{0}^{\infty} A k_{\mathrm{t}} \mathrm{e}^{-A \mathrm{t}} \mathrm{dt}=\mathrm{k}_{0}+\int_{0}^{\infty} \dot{k}_{\mathrm{t}} \mathrm{e}^{-A \mathrm{t}} \mathrm{dt},
$$

$$
\int_{0}^{\infty} A k_{\mathrm{t}} \mathrm{e}^{-A \mathrm{t}} \mathrm{dt}-\int_{0}^{\infty} \dot{k}_{\mathrm{t}} \mathrm{e}^{-A \mathrm{t}} \mathrm{dt}=\mathrm{k}_{0}
$$

Por lo tanto, de la ecuación (6), se sigue que

$$
\int_{0}^{\infty} c_{\mathrm{t}} \mathrm{e}^{-A} \mathrm{dt}=\mathrm{k}_{0}
$$


De esta manera,

$$
\mathrm{k}_{0}=\int_{0}^{\infty} \mathrm{C}_{\mathrm{t}} \mathrm{e}^{-A t} \mathrm{dt}=\frac{1}{\lambda_{0} \rho}
$$

En consecuencia,

$$
\frac{1}{\lambda_{0}}=\mathrm{k}_{0} \rho
$$

Por lo tanto, la trayectoria óptima de consumo satisface

$$
C_{t}=k_{0} \rho e^{(A-\rho)^{t}}
$$

donde $c_{0}=k_{0}$ ? . Claramente, esta trayectoria es un máximo (global), ya que $\ln \left(c_{t}\right)$ es una función cóncava. En la figura 1 se observa la trayectoria óptima del consumo como función de la tasa subjetiva de descuento ? y del producto marginal decapital $A$.

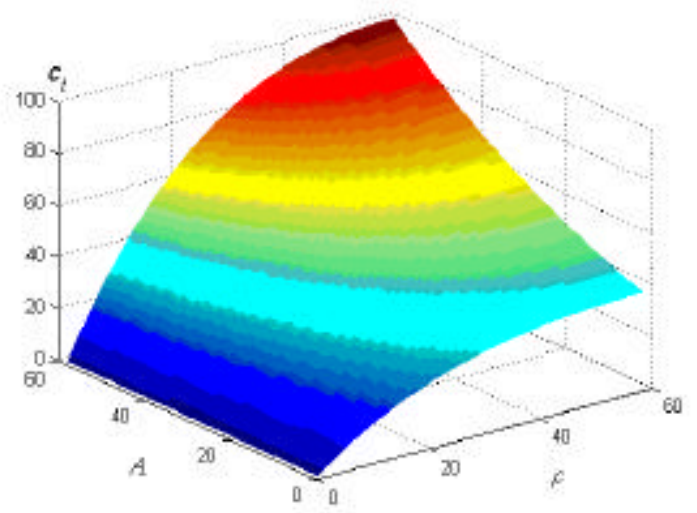

Figura 1

Por otro lado, se tiene que

$$
\dot{\mathrm{k}}_{\mathrm{t}}=A \mathrm{k}_{\mathrm{t}}-c_{\mathrm{t}}=A k_{t}-\mathrm{k}_{0} \rho \mathrm{e}^{(A-\rho)^{\mathrm{t}}}
$$

cuya solución, en términos del cap i tal, es

$$
\begin{gathered}
\mathrm{k}_{\mathrm{t}}=\mathrm{k}_{0} \mathrm{e}^{A t}-\mathrm{e}^{A} \int_{0}^{\mathrm{t}} k_{0} \rho \mathrm{e}^{(A-\rho) \mathrm{s}} \mathrm{e}^{-A s} \mathrm{ds} \\
=\mathrm{k}_{0} \mathrm{e}^{A \mathrm{t}}-\mathrm{e}^{A \mathrm{t}}\left(1-\mathrm{e}^{-\mathrm{d} t}\right)
\end{gathered}
$$

$$
=\mathrm{k}_{0} \mathrm{e}^{(A-\rho) t}
$$

En la figura 2 se observa la trayectoria óptima del

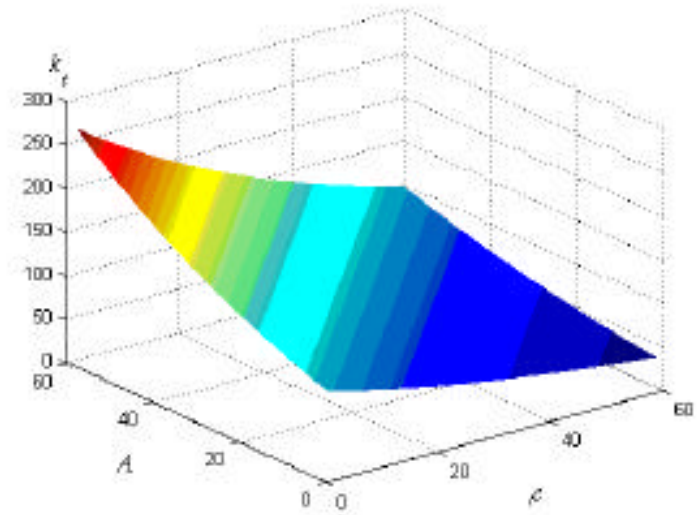

capital como función de la tasa ? y del producto marginal de cap ital $A$.

Figura 2

Ahora bien, como

$$
\begin{aligned}
\dot{\mathrm{c}} & =(A-\rho) \mathrm{c}_{\mathrm{t}} \\
\dot{\mathrm{k}}_{\mathrm{t}} & =(A-\rho) \mathrm{k}_{\mathrm{t}}
\end{aligned}
$$

se tiene que la tasa de crecimiento del consumo y la tasa de crecimiento del capital se encuentran respectivamente expresadas por las siguientes identidades

$$
\begin{aligned}
& \frac{\dot{\mathrm{c}}_{\mathrm{t}}}{\mathrm{c}_{\mathrm{t}}}=A-\rho, \\
& \frac{\dot{\mathrm{k}}_{\mathrm{t}}}{\mathrm{k}_{\mathrm{t}}}=A-\rho
\end{aligned}
$$

Asimismo, como $\mathrm{y}_{\mathrm{t}}=A \mathrm{k}_{\mathrm{t}}$, se tiene que $\dot{\mathrm{y}}_{\mathrm{t}}=A \dot{\mathrm{k}}_{\mathrm{t}}$. De lo an te rior, se concluye que

$$
\frac{\dot{\mathrm{y}}_{\mathrm{t}}}{\mathrm{y}_{\mathrm{t}}}=\frac{A \dot{\mathrm{k}} \mathrm{t}}{\mathrm{y}_{\mathrm{t}}}=\frac{A \dot{\mathrm{k}}_{\mathrm{t}}}{A \mathrm{k}_{\mathrm{t}}}=\frac{\dot{\mathrm{k}}_{\mathrm{t}}}{\mathrm{k}_{\mathrm{t}}}=A-\rho
$$

En consecuencia, el consumo, el capital y el producto crecen (o decrecen) exactamente a la misma tasa $A-\rho$. Si $A>\rho$, la producción crece, mientras que si $A<\rho$ la producción decrece. 
Cuando todos los sectores crecen (o decrecen) a la misma tasa se dice que el crecimiento en la economía es balanceado. En las figuras 3(a) y (c) se muestra el comportamiento del consumo y del capital respectivamente, cuando $A>\rho$. Asimismo, en las figuras $3(b)$ y (d) se muestra el compor-
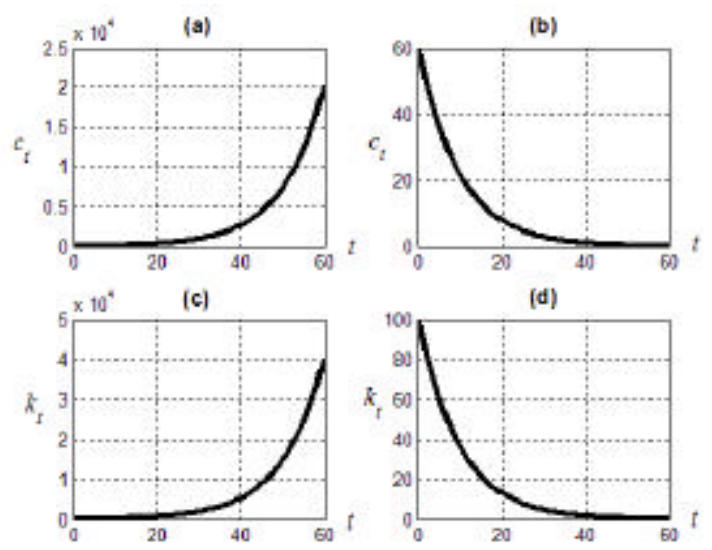

(d)

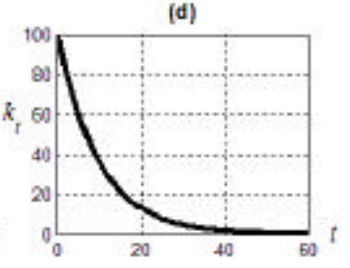

tamiento del consumo y del capital respectivamente, cuando $A<$ ? .

Figura 3

\section{Función de utilidad exponencial I}

Ahora, suponga que la función de utilidad del problema (2) está dada por

$$
u\left(c_{t}\right)=\frac{c_{t}^{\gamma}}{\gamma}
$$

Donde $k_{t} y c_{t}$ se definen como en el modelo anterior. El Hamiltoniano asociado a este problema se definecomo:

$$
H\left(k_{t}, c_{t}, \lambda_{t}\right)=\frac{c_{t}^{\gamma}}{\gamma}+\lambda_{t}\left(A k_{t}-c_{t}\right)
$$

donde ? es la vari able de coestado. A partir de la condición (4) se sigue que

$$
\dot{\lambda}_{\mathrm{t}}=\lambda_{\mathrm{t}}(\rho-A)
$$

Por lo tanto,

$$
\lambda_{t}=\lambda_{0} e^{(\rho-A)^{t}}
$$

La condición (3) con duce a

$$
c_{t}=\lambda_{t} \frac{1}{\gamma-1}
$$

y al sustituir ? ${ }_{t}$ en la ecuación an te rior, se tiene que

$$
G_{t}=\lambda_{0} \frac{1}{\gamma-1} e^{\frac{(\rho-A) t}{\gamma-1}}
$$

Ahora bien, como $c_{\mathrm{t}}=A \mathrm{k}_{\mathrm{t}}-\dot{\mathrm{k}}_{\mathrm{t}}$, se sigue que

$$
\mathrm{C}_{\mathrm{t}} \mathrm{e}^{-A t}=A k_{t} \mathrm{e}^{-A t}-\dot{k}_{t} \mathrm{e}^{-A t}
$$

Si se integra la expresión an te rior se obtiene

$$
\int_{0}^{\infty} c_{\mathrm{t}} \mathrm{e}^{-A t} \mathrm{dt}=\int_{0}^{\infty} A k_{t} \mathrm{e}^{-A t} \mathrm{dt}-\int_{0}^{\infty} \dot{k}_{t} \mathrm{e}^{-A t} \mathrm{dt}
$$

Observe que la integración por partes del primer sumando del lado derecho de la ecuación anteriorproduce

$$
\int_{0}^{\infty} A k_{\mathrm{t}} \mathrm{e}^{-A t} \mathrm{dt}=\mathrm{k}_{0}+\int_{0}^{\infty} \dot{k}_{t} \mathrm{e}^{-A t} \mathrm{dt}
$$

ó

$$
\int_{0}^{\infty} A k_{\mathrm{t}} \mathrm{e}^{-A t} \mathrm{dt}-\int_{0}^{\infty} \dot{k}_{t} \mathrm{e}^{-A t} \mathrm{dt}=\mathrm{k}_{0}
$$

Por lo tanto, de la ecuación (7), se sigue que

$$
\int_{0}^{\infty} c_{\mathrm{t}} \mathrm{e}^{-A t} \mathrm{dt}=\mathrm{k}_{0}
$$

De esta manera,

$$
\mathrm{k}_{0}=\int{ }_{0}^{\infty} \mathrm{C}_{\mathrm{t}} \mathrm{e}^{-A t} \mathrm{dt}=\lambda_{0} \frac{1}{\gamma-1}\left(\frac{\gamma-1}{\gamma A-\rho}\right)
$$

En consecuencia,

$$
\lambda_{0} \frac{1}{\gamma-1}=k_{0}\left(\frac{\gamma A-\rho}{\gamma-1}\right)
$$

Por lo tanto, la trayectoria óptima de consumo satisface

$$
c_{t}=k_{0}\left(\frac{\gamma A-\rho}{\gamma-1}\right) e^{\frac{(p-A) t}{\gamma-1}}
$$

donde $c_{0}=k_{0}[(? A-?) /(?-1)]$. En la figura 4 se observa la trayectoria óptima del consumo como función de la tasa ? y del producto marginal de capital $A$. 


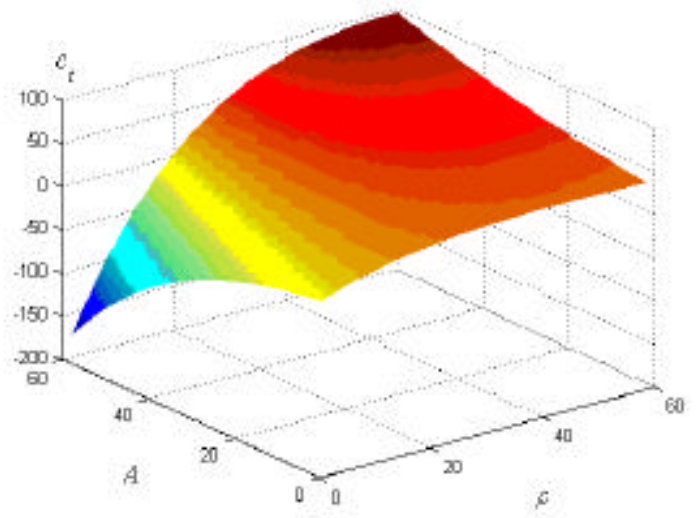

Figura 4

Por otro lado, se tiene que

$$
\dot{\mathrm{k}}_{\mathrm{t}}=A \mathrm{k}_{\mathrm{t}}-\mathrm{G}_{\mathrm{t}}=A \mathrm{k}_{\mathrm{t}}-\mathrm{k}_{0}\left(\frac{\gamma A-\rho}{\gamma-1}\right) \mathrm{e}^{\frac{(\rho-A) \mathrm{t}}{\gamma-1}}
$$

cuya solución en términos del cap i tal se encuentra dada por la siguiente expresión

$$
\begin{gathered}
k_{t}=k_{0} e^{A t}-e^{A t} \int_{0}^{t} k_{0}\left(\frac{\gamma A-\rho}{\gamma-1}\right) e^{\left(\frac{\rho-A}{\gamma-1}\right)} e^{-A s} d s= \\
k_{0} e^{\left(\frac{\rho-A}{\gamma-1}\right)}
\end{gathered}
$$

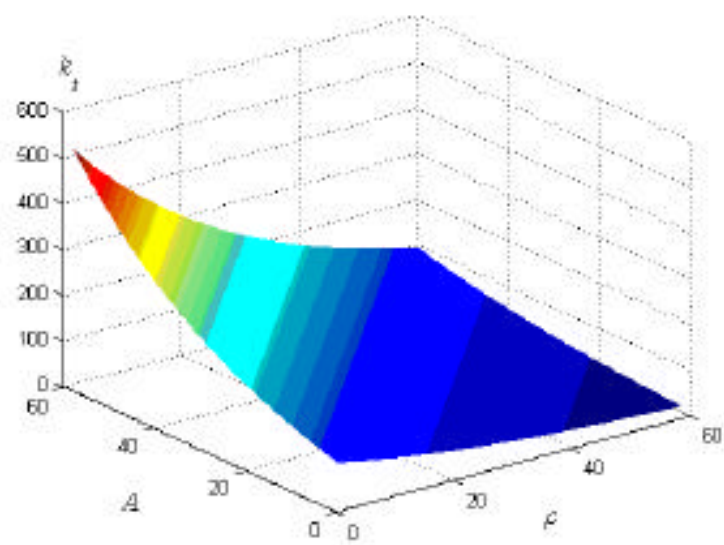

En la figura (5) se observa la trayectoria óptima de cap i tal como función de la tasa? y del producto marginal de cap i tal $A$.

Figura 5

Ahora bien, como

$$
\dot{\zeta}=\frac{\rho-A}{\gamma-1} c_{t}
$$

y

$$
\dot{\mathrm{k}}_{\mathrm{t}}=\left(\frac{\rho-A}{\gamma-1}\right)_{\mathrm{t}}
$$

Se tiene que la tasa de crecimiento del consumo y la tasa de crecimiento del capital, son expresadas respectivamente, por las siguientes ecuaciones

$$
\begin{aligned}
& \frac{\mathrm{G}_{\mathrm{t}}}{\mathrm{c}}=\left(\frac{\rho-A}{\gamma-1}\right) \\
& \frac{\dot{\mathrm{k}}_{\mathrm{t}}}{\mathrm{k}_{\mathrm{t}}}=\frac{\rho-A}{\gamma-1}
\end{aligned}
$$

Del mismo modo, como $\mathrm{y}_{\mathrm{t}}=A \mathrm{k}_{\mathrm{t}}$, se tiene que $\dot{y}_{t}=A \dot{k}_{t}$. De donde se concluye que

$$
\frac{\dot{\mathrm{y}}_{\mathrm{t}}}{\mathrm{y}_{\mathrm{t}}}=\frac{A \dot{\mathrm{k}}_{\mathrm{t}}}{\mathrm{y}_{\mathrm{t}}}=\frac{A \dot{\mathrm{k}}_{t}}{A k_{t}}=\frac{\dot{\mathrm{k}}_{\mathrm{t}}}{\mathrm{k}_{\mathrm{t}}}=\frac{\rho-A}{\gamma-1}
$$

En consecuencia, el consumo, el capital y el producto crecen (o decrecen) exactamente a la misma tasa $(?-A) /(?-1)$. Si $A>$ ?, la producción crece, mientras que si $A<$ ? la producción disminuye debido a que $?<1$. Cuando todos los sectores crecen (o decrecen) a la misma tasa se dice que el crecimiento en la economía es balanceado. En las figuras 6 (a) y (c) se muestra el comportamiento del consumo y del capital, respectivamente cuando $A>$ ?. Asimismo, las figuras 6 (b) y (d) muestran el comportamiento del consumo y del cap i tal, respectivamente cuando $A<$ ?. 

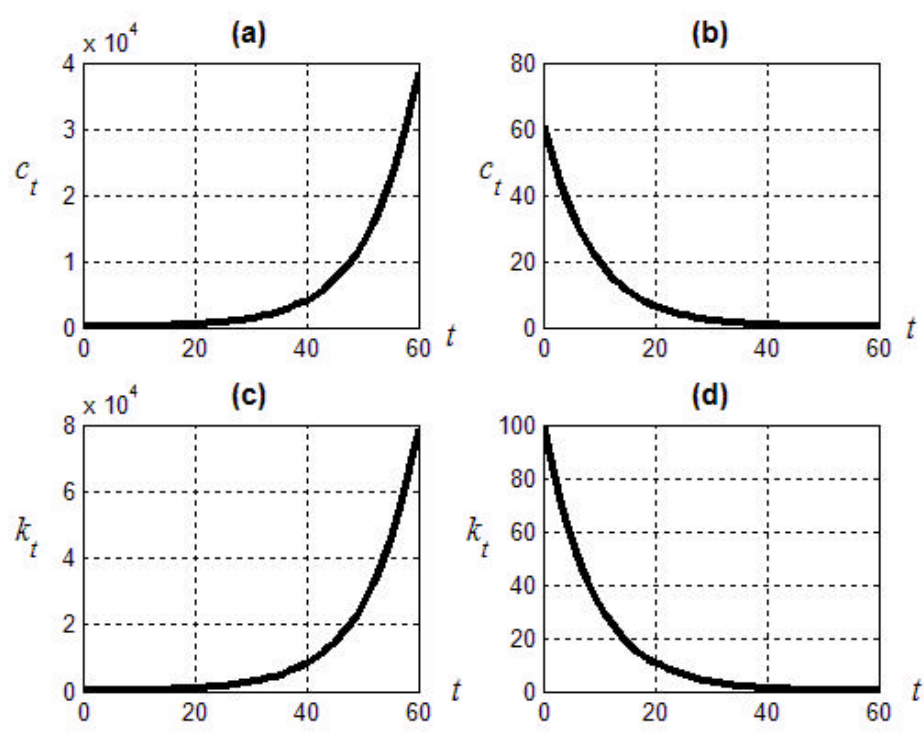

(d)

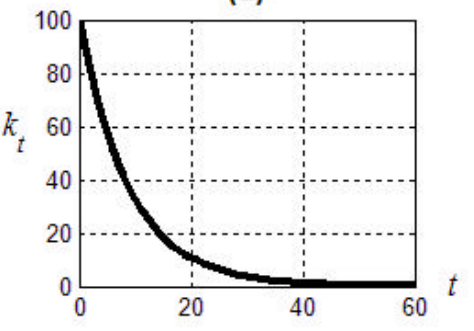

Figura 6

\section{Función de utilidad exponencial II}

Suponga ahora que la función de utilidad para el problema (2) se encuentra con la siguiente forma

$$
u\left(c_{t}\right)=\frac{c^{1-\theta}-1}{1-\theta}
$$

Donde $k_{t} y c_{t}$ se definen como en el modelo anterior. El Hamiltoniano asociado a este problema se definecomo:

$$
\mathrm{H}\left(\mathrm{k}_{\mathrm{t}}, \mathrm{C}_{\mathrm{t}}, \lambda_{\mathrm{t}}\right)=\frac{\mathrm{C}_{\mathrm{t}}^{1-\theta}-1}{1-\theta}+\lambda\left(A \mathrm{k}_{\mathrm{t}}-\mathrm{C}_{\mathrm{t}}\right)
$$

donde ?t es la vari able de coestado. A partir de la condición (4) se sigue que

$$
\dot{\lambda}_{\mathrm{t}}=\lambda_{\mathrm{t}}(\rho-A)
$$

Por lo tanto,

$$
\lambda_{t}=\lambda_{0} \mathrm{e}^{(\rho-A)}
$$

La condición (3) con duce a

$$
c_{\mathrm{t}}=\frac{1}{\lambda_{\mathrm{t}}^{1 / \theta}}
$$

Si se sustituye $?_{t}$ en la ecuación an te rior, se sigue que

$$
G_{t}=\lambda_{0}^{-1 / \theta} e^{\left(\frac{A-\rho}{\theta}\right)}
$$

Ahora bien, como $\mathrm{c}=A \mathrm{k}_{\mathrm{t}}-\dot{\mathrm{k}}_{\mathrm{t}}$, se tiene que

$$
c_{\mathrm{t}} \mathrm{e}^{-A t}=A k_{t} \mathrm{e}^{-A t}-\dot{\mathrm{k}}_{\mathrm{t}} \mathrm{e}^{-A t}
$$

Si se integra la expresión an te rior se obtiene

$$
\int_{0}^{\infty} c_{t} e^{-A t} d t=\int_{0}^{\infty} A k_{t} e^{-A t} \mathrm{~d}-\int_{0}^{\infty} \dot{k}_{t} \mathrm{e}^{-A t} \mathrm{dt}
$$

Observe que la integración por partes del primer sumando del lado derecho de la ecuación an teriorproduce

$$
\int_{0}^{\infty} A k_{t} \mathrm{e}^{-A t} \mathrm{dt}=\mathrm{k}_{0}+\int_{0}^{\infty} \dot{k}_{t} \mathrm{e}^{-A t} \mathrm{dt}
$$

ó

$$
\int_{0}^{\infty} A k_{t} \mathrm{e}^{-A t} \mathrm{dt}-\int_{0}^{\infty} \dot{k}_{t} \mathrm{e}^{-A t} \mathrm{dt}=\mathrm{k}_{0}
$$

Por lo tanto, de la ecuación (8), se tiene que

$$
\int_{0}^{\infty} c_{t} \mathrm{e}^{-A} \mathrm{dt}=\mathrm{k}_{0}
$$


De esta manera,

$$
\mathrm{k}_{0}=\int_{0}^{\infty} c_{\mathrm{t}} \mathrm{e}^{-A t} \mathrm{dt}=\lambda_{0}^{-\frac{1}{\theta}}\left(\frac{\theta}{\rho-A(1-\theta)}\right)
$$

En consecuencia,

$$
\lambda_{0}^{-1 / \theta}=k_{0}\left(\frac{\rho-A(1-\theta)}{\theta}\right)
$$

Por lo tanto, la trayectoria óptima de consumo satisface

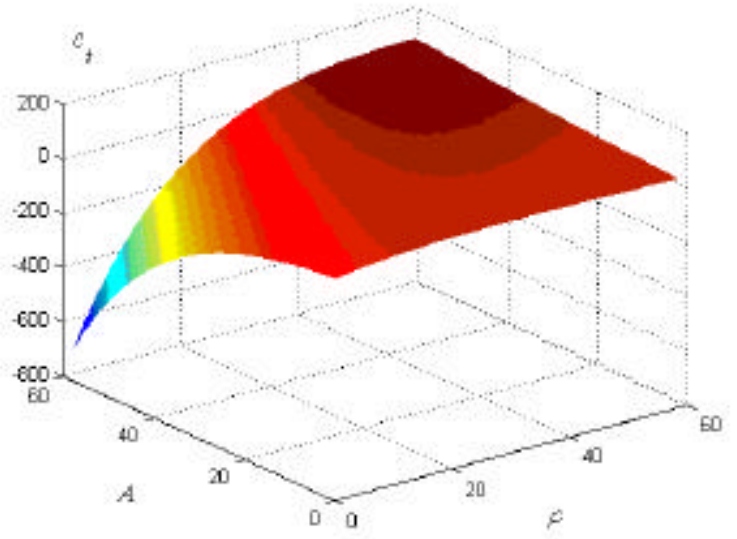

Figura 7

$$
c_{t}=k_{0}\left(\frac{\rho-A(1-\theta)}{\theta}\right) e^{\left(\frac{A-\rho}{\theta}\right) t}
$$

donde $c_{0}=\mathrm{k}_{0}[?-A(1-?)) /$ ?]. Ob serve en la figura 7 la trayectoria óptima del consumo como función de la tasa ? y del producto mar ginal de cap i tal $A$.

Por otro lado, se tiene que

$$
\dot{\mathrm{k}}_{\mathrm{t}}=A \mathrm{k}_{\mathrm{t}}-\mathrm{G}_{\mathrm{q}}=A \mathrm{k}_{\mathrm{t}}-\mathrm{k}_{0}\left(\frac{\rho-A(1-\theta)}{\theta}\right) \mathrm{e}^{\left(\frac{A-\rho}{\theta}\right)}
$$

cuya solución en términos del capitales

$$
\mathrm{k}_{\mathrm{t}}=k_{0} \mathrm{e}^{A t}-\mathrm{e}^{A t} \int_{0}^{\mathrm{t}} \mathrm{k}_{0}\left(\frac{\rho-A(1-\theta)}{\theta}\right) e^{\left(\frac{A-\rho}{\theta}\right) s} \mathrm{e}^{-A s} d s
$$

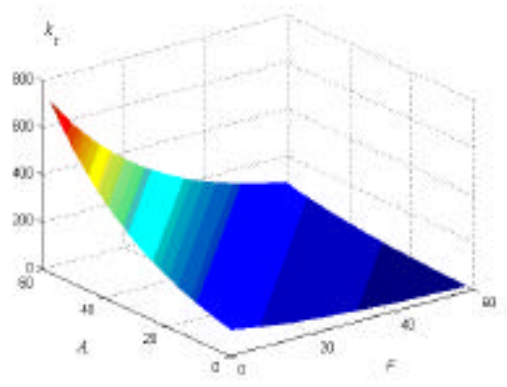

$$
=k_{0} e^{\left(\frac{A-\rho}{\theta}\right) t}
$$

En la figura 8 se observa la trayectoria óptima del capital como función de la tasa ? y del producto mar ginal de cap i tal $A$.

Figura 8

Ahora bien, como

$$
\begin{array}{r}
\dot{\mathrm{c}}_{\mathrm{t}}=\left(\frac{A-\rho}{\theta}\right) \mathrm{c}_{\mathrm{t}} \\
\dot{\mathrm{k}}_{\mathrm{t}}=\left(\frac{A-\rho}{\theta}\right) \mathrm{k}_{\mathrm{t}}
\end{array}
$$

Se tiene que la tasa de crecimiento del consumo y la tasa de crecimiento del capital se encuentran expresados respectivamente, de la siguiente manera

$$
\begin{aligned}
& \frac{\dot{\mathrm{c}}}{\mathrm{c}_{\mathrm{c}}}=\frac{A-\rho}{\theta} \\
& \frac{\dot{\mathrm{k}_{\mathrm{t}}}}{\mathrm{k}_{\mathrm{t}}}=\frac{A-\rho}{\theta}
\end{aligned}
$$

Del mismo modo, como $\mathrm{y}_{\mathrm{t}}=A \mathrm{k}_{\mathrm{t}}$, se tiene que $\mathrm{yt}=\mathrm{A} \dot{\mathrm{k}}_{\mathrm{t}}$. De donde se concluye que

$$
\frac{\dot{\mathrm{y}}_{\mathrm{t}}}{\mathrm{y}_{\mathrm{t}}}=\frac{A \dot{\mathrm{k}}_{\mathrm{t}}}{\mathrm{y}_{\mathrm{t}}}=\frac{A \dot{k}_{t}}{A k_{\mathrm{t}}}=\frac{\dot{k}_{\mathrm{t}}}{\mathrm{k}_{\mathrm{t}}}=\frac{A-\rho}{\theta}
$$

En consecuencia, el consumo, el capital y el producto crecen (o decrecen) exactamente a la misma tasa [( $A-$ ? $) /$ ?]. Si $A>$ ?, la producción crece, mientras que si $A<$ ? la producción disminuye debido a que ? $>0$. Cuando todos los sectores 

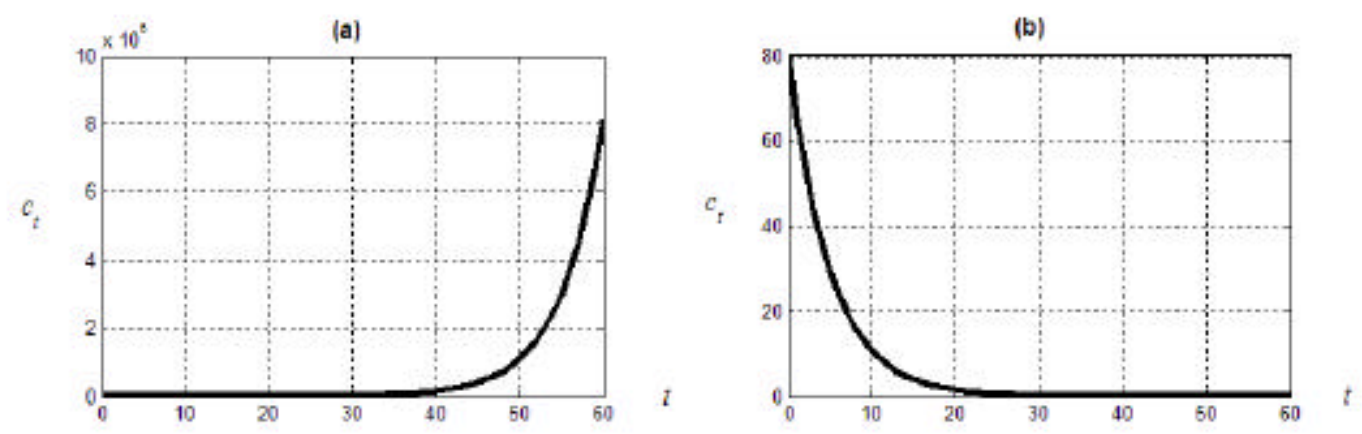

(c)
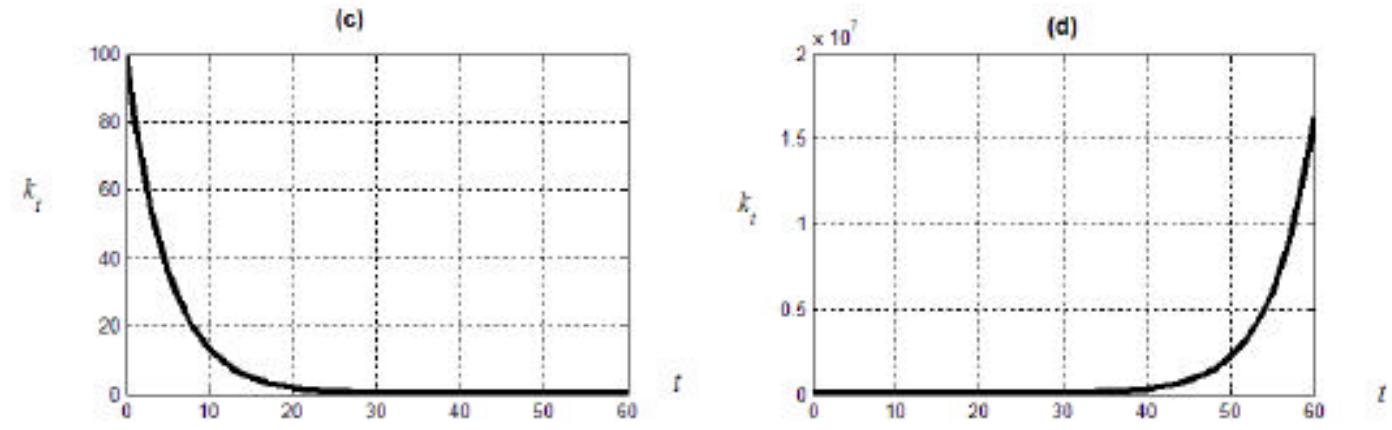

Figura 9

crecen (o decrecen) a la misma tasa se dice que el crecimiento en la economía es balanceado. En las figuras 9 (a) y (c) se muestra el comportamiento del consumo y del capital respectivamente, cuando $A>$ ?. Asimismo las figuras 9 (b) y (d) muestran el comportamiento del consumo y del capital respectivamente cuando $A<$ ?

\section{Función de utilidad exponencial negativa}

Considere la siguiente ecuación de utilidad para el problema (2)

$$
u\left(c_{t}\right)=-e^{-\theta c_{t}}
$$

En el marco de la teoría de con trol óptimo, $k_{t}$ es la vari able de estado y $c_{t}$ es la variable de control. El Hamiltoniano asociado a este problema se define como:

$$
H\left(k_{t}, c_{t} \lambda_{t}\right)=-e^{-\theta c_{t}}+\lambda_{t}\left(A k_{t}-G_{t}\right)
$$

donde ${ }_{t}$ es la vari able de coestado. La condición (3) con duce a:

$$
\theta e^{-\theta c_{t}}-\lambda_{t}=0
$$

es decir

$$
c_{t}=\frac{h(\theta)-\ln \left(\lambda_{t}\right)}{\theta}
$$

y al sustituir ? en la ecuación an te rior, se sigue que

$$
c_{\mathrm{t}}=\frac{\ln (\theta)-\ln \left(\lambda_{0}\right)-(\rho-A) t}{\theta}
$$

Ahora bien, como $c_{t}=A k_{t}-\dot{k}$, se tiene que

$$
c_{\mathrm{t}} \mathrm{e}^{-A t}=A k_{t} \mathrm{e}^{-A t}-\dot{\mathrm{k}}_{\mathrm{t}} \mathrm{e}^{-A t}
$$

Si se integra la expresión an te rior se obtiene

$$
\begin{gathered}
\int{ }_{0}^{\infty} \mathrm{C}_{\mathrm{t}} \mathrm{e}^{-A t} \mathrm{dt}=\int_{0}^{\infty} A k_{t} \mathrm{e}^{-A t} \mathrm{dt} \\
-\int{ }_{0}^{\infty} \dot{\mathrm{k}}_{\mathrm{t}} \mathrm{e}^{-A t} \mathrm{dt}
\end{gathered}
$$


Observe que la integración por partes del primer sumando del lado derecho de la ecuación anteriorproduce

ó

$$
\int_{0}^{\infty} A k_{t} \mathrm{e}^{-A t} \mathrm{dt}=\mathrm{k}_{0}+\int_{0}^{\infty} \dot{\mathrm{k}}_{\mathrm{t}} \mathrm{e}^{-A t} \mathrm{dt}
$$

$$
\int_{0}^{\infty} A k_{t} \mathrm{e}^{-A t} \mathrm{dt}-\int_{0}^{\infty} \dot{\mathrm{k}}_{\mathrm{t}} \mathrm{e}^{-A t} \mathrm{dt}=\mathrm{k}_{0}
$$

De esta manera, de la ecuación (9), se tiene que

$$
\int_{0}^{\infty} c_{t} \mathrm{e}^{-A t} \mathrm{dt}=\mathrm{k}_{0}
$$

De donde se sigue que

$$
\mathrm{k}_{0}=\int_{0}^{\infty} c_{t} \mathrm{e}^{-A t} \mathrm{dt}=\frac{1}{\theta}\left[\frac{\ln (\theta)-\ln \left(\lambda_{0}\right)}{A}-\frac{(\rho-A)}{A^{2}}\right]
$$

En consecuencia,

$$
\ln (\theta)-\ln \left(\lambda_{0}\right)=A \theta k_{0}+\frac{\rho-A}{A}
$$

Por lo tanto, la trayectoria óptima de consumo satisface

$$
C_{\mathrm{t}}=\frac{A \theta \mathrm{k}_{0}+\frac{\rho-A}{A}-(\rho-A) t}{\mathrm{~A}}
$$

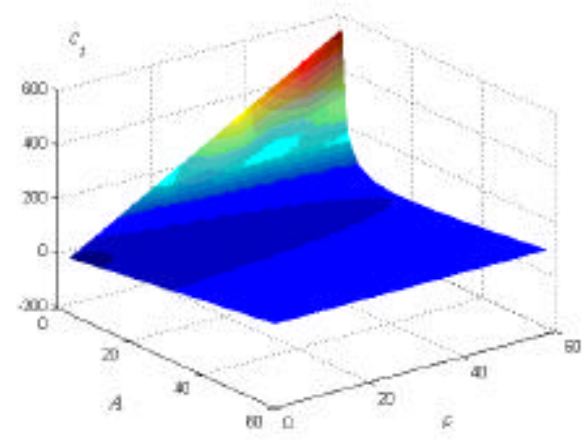

donde

$$
\sigma_{0}=\left(A \theta \mathrm{k}_{0}+\frac{\rho-A}{A}\right) / \theta
$$

Ob serve en la figura 10 la trayectoria óptima del consumo como función de la tasa ? y del producto marginal de cap ital $A$.

Figura 10
Por otro lado, se tiene que

$$
\dot{\mathrm{k}}_{\mathrm{t}}=A \mathrm{k}_{\mathrm{t}}-\mathrm{c}_{\mathrm{t}}=A \mathrm{k}_{\mathrm{t}}-\left(\frac{A \theta k_{0}+\frac{\rho-A}{A}-(\rho-A) t}{\theta}\right)
$$

cuya solución en términos del capital $k_{t}$ es $\mathrm{k}_{0} \mathrm{e}^{A \mathrm{t}}$

$$
e^{A t} \int_{0}^{\mathrm{t}}\left(\frac{A \theta k_{0}+\frac{\rho-A}{A}-(\rho-A) s}{\theta}\right) e^{-A s} d s
$$

De donde, $k_{t}=k_{0}+((A-?) / A$ ?)t. En la figura 11 se observa la trayectoria óptima del capital como función de la tasa ? y del producto marginal de capital $A$.

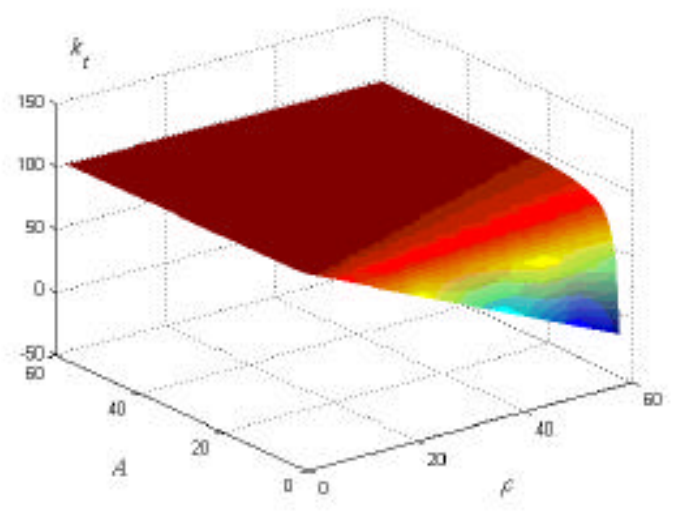

Ahora bien, como

$$
\frac{\dot{y}_{t}}{y_{t}} \neq \frac{c_{t}}{c_{t}} \neq \frac{\dot{k}_{t}}{k_{t}}
$$

se concluye que, para este modelo, no es posible que el crecimiento en la economía sea balanceado.

Figura 11

\section{Conclusiones}

Este trabajo desarrolló, dentro de un marco determinista, un modelo de crecimiento económico que explica de forma endógena los determinantes del 
mismo. Este problema considera aspectos como, consumo, producción e inversión. Asimismo, el modelo propuesto de scribe el comportamiento de la dinámica de las trayectorias de consumo y cap ital de los agentes. En el planteamiento del problema se supuso que el producto mar ginal del capital se mantiene constante en el tiempo. Por ello, se planteó el problema de decisión de un consumidor, que desea maximizar su utilidad por un bien de consumo perecedero, como un problema de con trol óptimo determinista en tiempo con tinuo. Se revisaron distintas funciones de utilidad en las que se examinaron las decisiones óptimas del consumidor utilizando subrutinas de MATLAB (c). Un aspecto interesante de este problema es que para los modelos con funciones de utilidad logarítmica y exponencial se encontró que el crecimiento en la economía es balanceado, debido a que el consumo, el capital y el producto evolucionan exactamente a la misma tasa. En cambio, para el modelo con una función de utilidad exponencial negativa se concluye que no es posible que el crecimiento en la economía sea balanceado.

\section{Referencias}

Cerdá E. (2001). O ptimización dinámica. Ed. Prentice Hall.

Chiang A. (1992). Dynamic Optimization. McGraw-Hill.

\section{Bibliografía sugerida}

Bellman R. (1957). Dynamic Programming. Princeton Univer sity Press.
Bellman R. (1961). Adaptive Control Processes: A Guided Tour. Princeton Univer sity Press.

Bertsekas Dimitri P. (1987). Dynamic Programming: Deterministic and Stochastic M odels . Prentice Hall.

Chiang A. (1987). Métodos fundamentales de economía matemática. $3 a$ Edición, McGraw-Hill.

Dorfman, R. (1969). An Economic Interpretation of Optimal Control Theory. American E conomic Review, No. 59, pp. 817-831.

Fleming W. y Rishel R. (1975). Deter min isticand Stochastic 0 ptimal Control. Springer-Verlag.

Gelfand I.M. y Fomin S.V. (1963). Calculus of Variations. Prentice-Hall.

Venegas-Martinez F. (1999). Crecimiento endógeno, dinero, impuestos y deuda externa. Investigación económica, Facultad de Economía, UNAM, Vol. 59, No. 229, pp.15-36.

Venegas-Martinez F. (2000). On consump tion, Investment and Risk. Economía Mexicana, Nueva Época, División de Economía, CIDE, 9, No. 2, pp.227-244.

Venegas-Martinez F. (2000). Utilidad, aprendizaje y estabilización. Gaceta de E conomía, ITAM, Año 5, No. 10, pp.153-169.

Venegas-Martinez F. (2001). Tempo rary Stabi lization: A Stochastic Analysis, Journal of Economic Dynamics and Control, Vol. 25, No. 9, September, pp.1429-1449.

Weber Richard (2002). Optimization and Control. Http://www.statslab.com.ac.uk/ rrw1/oc /index.html

\section{Semblanza de los autores}

Víctor Manuel García-Guerrero. Obtuvo la maestría en investigación de operaciones y la licenciatura en actuaría en la UNAM. Imparte cátedra en la Facultad de Ciencias de la UNAM y es miembro de la International Society on Multiple Criteria Decision Making desde 2003. Actualmente se desempeña como asesor de auditoría, metodología y administración de riesgos de crédito en BBVA Bancomer.

Francisco Venegas-M artínez. Labora en el Tecnológico de Monterrey, Campus Ciudad de México como director del Centro de Investigación en Finanzas, director de la maestría y doctorado en ciencias financieras y como editor de la Revista Mexicana de Economía y Finanzas. Tiene estudios de postdoctorado en finanzas en 0 xford Univer sity, un doctorado en matemáticas y otro en eco no mía por la Wash ington State Univer sity, la maestría en econo mía en el ITAM, la maestría en matemáticas y la de investigación de operaciones en la UNAM. Ha sido profesor-investigador en la UNAM, UAM, UP, Washington State University, CIDE, Oxford University y Tecnológico de Monterrey. Asimismo, ha sido profesor en el COLMEX en la Universidad Anáhuac y en el Colegio de la Frontera del Norte. Fue ganador del Premio Nacional en Investigación Económica "Maestro Jesús Silva Herzog 2002" y del premio Nacional "Mex Der 2003". 